ORIGINAL ARTICLE

\title{
Non-touch suturing technique fails to reduce glove puncture rates in an accident and emergency department
}

\author{
T K McAdam, R E McLaughlin, B McNicholl
}

Emerg Med J 2004;21:560-561. doi: 10.1136/emj.2002.001461

See end of article for
authors' affiliations
......................
Correspondence to:
Mr B McNicholl,
Emergency Department,
Royal Victoria Hospital,
Grosvenor Road, Belfast
BT12 6BA, UK;
brian.mcnicholl@
royalhospitals.n-i.nhs.uk
Accepted for publication
1 April 2003
.........................

\begin{abstract}
Objective: To assess the impact of introducing a safer non-touch suturing technique into an inner city emergency department.

Methods: The rate of glove perforation, measured by electrical conductance, was used as a marker. Gloves (Bodyguards) used in suturing were collected over a two month period. Two half day suture workshops were then conducted in the emergency department and gloves were collected for a further two months. All doctors (14) were included in the study regardless of grade. Gloves were tested for perforation by electrical conductance.

Results: There were 107 gloves and 19 perforations in the first group, 133 with 28 perforations in the second $(p=0.52)$, and one perforation in 100 control gloves. Most doctors reported inadvertent needlestick handling.

Conclusion: Two half day suturing workshops are not enough to reduce glove perforations from suturing in the emergency department. Difficulty with compliance suggests that safe suturing practice must be taught before doctors develop bad habits that are difficult to change.
\end{abstract}

$\mathrm{S}$ urgical gloves provide a mechanical barrier against communicable disease. ${ }^{12}$ Pooled data indicate the average probability of transmission after needlestick exposure is $0.2 \%-0.5 \%$ for HIV- $1,30 \%$ for hepatitis B, and between $5 \%$ and $10 \%$ for hepatitis $\mathrm{C}^{3}$ A recent study from Sheffield highlighted deficiencies in the teaching of practical skills and use of universal precautions to promote sharps safety for medical students and junior doctors. ${ }^{4}$ To improve sharps practice in our emergency department we trained all our doctors in non-touch suturing techniques as taught by the Royal College of Surgeons basic surgical skills course. This study assessed the impact of the training by measuring glove perforation rates after suturing. The training and techniques were then assessed by questionnaire.

\section{METHODS}

Over a four month period, in an inner city emergency department with 50000 new patients per annum, all gloves used by doctors for suturing were collected. Suturing was performed using standard packs with nylon (Ethicon) suture material and latex (Bodyguards) gloves. Participants included three consultants, three middle grade staff, and eight senior house officers.

Gloves were collected in bins marked dominant and nondominant. After two months, when the senior house officers and two of the registrars changed posts, an in-house suture workshop was undertaken for all senior house officers, middle grade staff, and consultants. They were taught a dedicated non-touch technique (NTT) in accordance with the basic surgical skills course. All managed to perform it successfully. Two weeks later another similar workshop was held to reinforce the technique.

The electrical conductance test ${ }^{5}$ was used to test for glove perforations. (A standard ohmmeter (Caltec category 2 CM 1100 ) will show a decrease in electrical resistance if there is a hole in the latex, using saline inside and outside the glove as a conducting medium). After the training and testing phases of this study all doctors were then sent a questionnaire regarding their views of the training provided and the value of the NTT.

\section{RESULTS}

A perforation rate of $17.78 \%$ ( 19 of 107) occurred in the preintervention group and a rate of $18.7 \%$ (28 of 150) in the post-intervention group $\left(p=0.52, \chi^{2}\right)$. A control of 100 unused gloves produced a perforation rate of $1 \%$ ( 1 of 100), below the industry standard of $1.5 \% .{ }^{6}{ }^{7}$ Table 1 shows the rate of perforations in the dominant and non-dominant hands before and after intervention.

Fourteen questionnaires were sent and 12 returned (86\%). Nine doctors (75\%) made an effort to use NTT; four doctors (33\%) expressed difficulty changing to NTT. Eleven (92\%) admitted to inadvertently handling the needle during suturing. Most felt that NTT was worthwhile (11, 92\%) and that the training was adequate $(8,76 \%)$, but nine doctors $(75 \%)$ said they would like further training. Five doctors (42\%) admitted to 12 needlestick injuries during this study.

\section{DISCUSSION}

Despite two workshops teaching a recommended NTT the perforation rate in gloves did not change between the groups. The likeliest explanation is difficulty changing to a new technique and inadvertent needle handling ( 11 of $12,92 \%$ ) as indicated by the questionnaire. It is unlikely that any one member of staff with a high puncture rate could have biased the results as most of the staff changed over and the volume of suturing performed by the remaining staff was comparatively small.

There is no research showing that NTT can effectively reduce needlestick injury or glove perforations in emergency departments. A reduction in glove perforation using NTT during abdominal wound closure has been shown in theatre. $^{89}$ However, there were no compliance problems reported or breach of protocol in these studies. It may be easier to introduce and enforce a safe suturing technique in a theatre environment where team members are all aware of the research and reinforcing the technique with each other. In contrast, doctors in the emergency department usually suture without supervision by other doctors or a research team and needlestick handling was noted during the study. 
Table 1 Pattern of glove perforations before and after the intervention of a suture workshop

\begin{tabular}{|c|c|c|}
\hline & $\begin{array}{l}\text { Perforation rate (\%) } \\
\text { before workshop }\end{array}$ & $\begin{array}{l}\text { Perforation rate (\%) } \\
\text { after workshop }\end{array}$ \\
\hline $\begin{array}{l}\text { Dominant hand } \\
\text { Non-dominant hand } \\
\text { Overall perforation rate }\end{array}$ & $\begin{array}{l}14.8 \\
20.8 \\
17.8\end{array}$ & $\begin{array}{l}21.8 \\
14.3 \\
18.7\end{array}$ \\
\hline
\end{tabular}

We did not use needlestick injury as a measure of effectiveness, as reporting is known to be unreliable. ${ }^{11}{ }^{12}$ Glove perforations with significant contamination can remain unnoticed in up to $83 \%$ of surgeons. ${ }^{1}$ There were no reported needlestick injuries to occupational health during this study despite $42 \%$ of our doctors admitting needlestick injuries. Trust policy requires the reporting of all needlestick injuries to the occupational health department.

Suggested measures to aid prevention of glove perforation in suturing include; no touch technique, ${ }^{9}$ double gloving, ${ }^{1}$ green undergloves, ${ }^{13}$ and safe sharps handling.

A comparative study of glove perforation rates after suturing in an emergency department reports a rate of $5.4 \%$ per glove. ${ }^{14}$ Previous research with hollow needles used for intravenous access in our department ${ }^{10}$ shows a perforation rate of $6.9 \%$ in the dominant hand suggesting that simple manual tasks with the same gloves in our department does not lead to a high perforation rate.

Our higher rate may be explained in a number of ways. This study used the electrical conductance test for detection of perforations whereas the other studies used the water load test, which is less sensitive. ${ }^{5}$ It could also be argued that with the advent of tissue glues, staples, and nurse practitioners, junior doctors are not receiving adequate practice in basic suturing. They are likely to suture more complex wounds, which take longer, are more technically difficult, and may be associated with a higher glove perforation rate.

Some doctors $(33 \%)$ found it difficult to change their practice to a NTT. This emphasises the need to teach it at undergraduate level. Some specialties such as general surgery have mandatory courses that basic surgical trainees are encouraged to take early in their training. ${ }^{15}$ This should be applied to all specialties where suturing is performed. There is evidence that doctors infrequently performing sharps tasks have increased rates of needlestick injury, ${ }^{16}$ all the more reason to include this training at undergraduate level.

Training in NTT did not reduce glove perforation rates in our emergency department probably because of poor compliance, although the NTT has not been proved to prevent glove perforations completely. Further research is required to validate the technique and explore the reasons for poor compliance.

Of greater concern is that despite two workshops, glove perforations and needlestick injuries did occur The annualised rate for these are 11 glove perforations and five detected needlestick injuries per doctor per year from suturing.

Some doctors indicated they would like more training. This needs to be explored. The philosophy of the NTT is simple (do not handle the needle with your fingers) and the technique comparatively easily performed (doctors successfully managed it during workshops). It is not clear what additional training should include. Our doctors received more teaching time on this subject than any other induction topic including cardiopulmonary resuscitation and other critical interventions. If we devote more time to suturing techniques this would be at the expense of other, perhaps more important subjects that our critical incident monitoring highlight. In our current teaching programme suturing usually gets one workshop session. We gave it two this time. We will not justifiably be able to run three workshops to give our doctors more training as they suggested, within our resource framework. It is therefore unlikely that our next team of doctors will fare any better in terms of safety. This emphasises the need to look at medical school training and other techniques to improve safety. Perhaps learning non-touch techniques, which are of confirmed benefit, ${ }^{17}$ at the start of medical school suturing would be sustained. Our doctors found it difficult to change unsafe techniques. It would be of great concern if this problem pertains to practising doctors in other emergency departments or surgical disciplines.

\section{Authors' affiliations}

T K McAdam, R E McLaughlin, B McNicholl, Emergency Department, Royal Victoria Hospital, Belfast, UK

\section{REFERENCES}

1 Thomas S, Agarwal M, Mehta G. Intraoperative glove perforation-single versus double gloving in protection against skin contamination. Postgrad Med J 2001;77:458-60.

2 Dalgleish AG, Malkovsky M. Surgical gloves as a mechanical barrier against human immunodeficiency viruses. Br J Surg 1988;75:171-2.

3 Mejicano GC, Maki DG. Infections acquired during cardiopulmonary resuscitation: estimating the risk and defining strategies for prevention. American Society of Internal Medicine 1998;129:813-28.

4 Goodfellow PB, Claydon P. Students sitting medical finals-ready to be house officers? J R Soc Med 2001;94:516-20.

5 Sohn RL, Murray MT, Franko A, et al. Detection of surgical glove integrity. Am Surg 2000;66:302-6.

6 ASTM committee D-11. Sub-committee D1 1.40 on consumer rubber products. Standard test method for detection of holes in medical gloves. D5151-92:171-172.

7 ASTM committee D-11. Sub-committee D1 1.40 on consumer rubber products. Standard test method for detection of holes in medical gloves. D3700-00:1-4.

8 Chan $P$, Lewis AAM. Influence of suture technique on surgical glove perforation. Br J Surg 1989;76:1208-9.

9 Corlett MP, England DW, Kidner NL, et al. Reduction in incidence of glove perforation during laparotomy wound closure by "no touch" technique. Ann R Coll Surg Engl 1993;75:330-2.

10 Mc Adam TK, Mc Laughlin RE, Mc Nicholl B. Needlestick injuries-are we getting the point? Int J STD AIDs (in press).

11 Hamory BH. Underreporting of needlestick injuries in a university hospital. Am J Infect Control 1983;11:174-7.

12 Tandberg D, Stewart KK, Doezema D. Under-reporting of contaminated needlestick injuries in emergency health care workers. Ann Emerg Med 1991;20:66-70.

13 Brown JN. Surgeon protection: early recognition of glove perforation using a green under glove. J R Coll Surg Edinb 1996;41:395-6.

14 Hansen KN, Korniewicz DM, Hexter DA, et al. Loss of glove integrity during emergency department procedures. Ann Emerg Med 1998;31:1.

15 Royal College of Surgeons of Edinburgh. Information for courses. (http:// www.rcsed.ac.uk/education/bss/BSSdetails.asp)

16 Patel N, Tignor GH. Device specific sharps injury and usuage rates: an analysis by hospital department. Am J Infect Control 1997;25:77-84.

17 MCLaughlin REMC, MCAdam TK, Sillery E, et al. The influence of the basic surgical skills course on needlestick injuries. Br J Surg 2002;89(suppl):78. 